\title{
Colossal Paramagnetic Moments in Metallic Carbon Nanotori
}

\author{
Lei Liu, ${ }^{1}$ G. Y. Guo, ${ }^{2}$ C. S. Jayanthi, ${ }^{1}$ and S. Y. Wu ${ }^{1}$ \\ ${ }^{1}$ Department of Physics, University of Louisville, Louisville, Kentucky 40292 \\ ${ }^{2}$ Department of Physics, National Taiwan University, Taipei, Taiwan 106
}

(Received 30 October 2001; published 14 May 2002)

\begin{abstract}
Carbon nanostructures with unusually large paramagnetic moments have been discovered in a theoretical study of the electronic and magnetic properties of carbon nanotubes bent into toroids. Specifically, nanotori formed from metallic nanotubes with $\lambda_{F}=3 T$, where $\lambda_{F}$ is the Fermi wavelength and $T$ the translation vector of the nanotube, exhibit giant paramagnetic moments at selected radii ("magic radii"), while the ones with $\lambda_{F}=T$ are paramagnetic at any radius. The large paramagnetic moment is due to the interplay between the toroidal geometry and the ballistic motion of the $\pi$ electrons in the metallic nanotube.
\end{abstract}

PACS numbers: $75.75 .+\mathrm{a}, 73.22 .-\mathrm{f}$

The search for carbon nanostructures with large magnetic moments has been pursued actively ever since Kroto et al. [1] speculated a large diamagnetic response for $\mathrm{C}_{60}$. However, it was shown both theoretically and experimentally that $\mathrm{C}_{60}$ would exhibit a very small magnetic susceptibility [2-4]. Experimental measurements also yielded that the (orientationally averaged) magnetic susceptibility of a carbon nanotube is about $-25 \mathrm{ppm} \mathrm{emu/g}[5,6]$, while that of graphite is about $-30 \mathrm{ppm} e \mathrm{mu} / \mathrm{g}$ for a magnetic field along the $c$ axis [6]. Recently, there have been reports on the observation of ring-shaped carbon nanostructures [7-10]. Because of the geometry of nanotori, they appear to be ideal candidates for enhancing the effect of the interplay between the delocalized $\pi$ electrons and the geometrical structure of the torus, and thus one may anticipate an enhanced magnetic response of these systems in the presence of an applied magnetic field.

In the present work, we have investigated the magnetic response of carbon nanotori under an applied magnetic field (with the field perpendicular to the plane of tori) based on a $\pi$-orbital tight-binding theory. We found that, although not all nanotori formed from metallic nanotubes are metallic, those that are metallic exhibit a surprising 3 orders of magnitude larger paramagnetic moments compared to the diamagnetic moment of graphite at $0 \mathrm{~K}$ and at applied magnetic fields of $\sim 0.1 \mathrm{~T}$. Our investigation has also elucidated succinctly the physics underpinning the unusual magnetic response of nanotori as due to the interplay between the toroidal geometry and the $\pi$-electron dominated electronic structure.

The $\pi$-orbital tight-binding Hamiltonian has been successfully applied to study the electronic structure of $\mathrm{C}_{60}$-based materials and nanotubes [11], in particular magnetic properties of nanotubes [12]. The effect of the magnetic field enters the Hamiltonian via $H_{i j}=H_{i j}^{0} \exp \left\{i(2 \pi / \phi) \int_{i}^{j} \mathbf{A} \cdot d \mathbf{r}\right\}$, where $H_{i j}^{0}$ is the Hamiltonian matrix element for zero field, $\phi=h c / e$ the flux quantum, and $\mathbf{A}$ the vector potential $[13,14]$. The magnetic moment of a nanotorus was calculated in terms of the ring current $I$ in the torus, namely,
$M=I \sigma$ where the current element between sites $i$ and $j$ is $I_{i j}=(4 e / \hbar) \operatorname{Im} \Sigma_{n} f\left(E_{n}\right) c_{i n}^{*} H_{i j} c_{j n}$ with $f\left(E_{n}\right)$ being the Fermi factor, $c_{i n}$ the eigenvector corresponding to the eigenenergy $E_{n}$, and $\sigma$ the area enclosed by the torus.

The induced magnetic moment vs temperature for a wide variety of nanotori that are formed from nanotubes of different chiralities (arm-chair, chiral, and zigzag) exhibiting both semiconducting and metallic behaviors is shown in Fig. 1 while the ring current structures are shown in Fig. 2. As can be seen from Fig. 1, a clear pattern has emerged for the magnetic response of nanotori with one set of nanotori exhibiting a diamagnetic response and the other a paramagnetic response. Specifically, we note the following outstanding features: (i) Nanotori formed from metal-1 nanotubes (i.e., those with the band degeneracy at $k=0$ [11]) possess unusually large paramagnetic moments at $0 \mathrm{~K}$. For example,

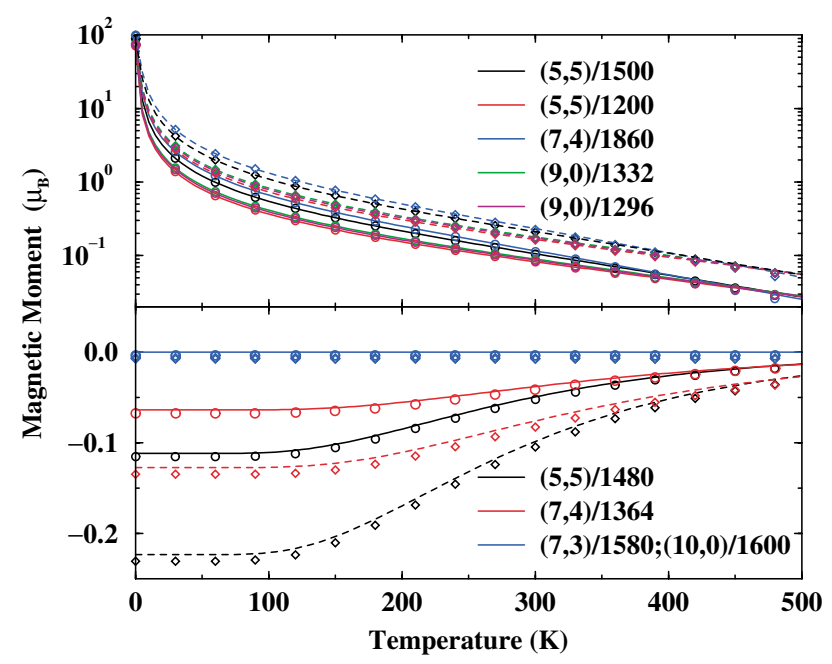

FIG. 1 (color). Induced magnetic moment vs temperature of various carbon nanotori (metallic as well as semiconducting tori) in a perpendicular magnetic field of $0.1 \mathrm{~T}$ (solid line) and $0.2 \mathrm{~T}$ (dashed line), respectively, as calculated from the ring currents using $M=I \sigma$. Circles and diamonds denote the magnetic moments calculated for the same set of nanotori using Eq. (1) at $B=0.1$ and $B=0.2 \mathrm{~T}$, respectively. 



FIG. 2 (color). Geometric and ring current structures: (a) the geometric structure of $(5,5) / 1200$ metallic nanotorus and the corresponding ring current; (b) the geometric structure of $(7,4) / 1364$ semiconducting nanotorus and the corresponding ring current. The colored arrows denote the ring currents with their lengths proportional to the current strength. The current flow follows exactly the chirality of the geometric structure of the torus. The applied magnetic field is directed towards the reader. The color coding has no significance other than providing guidance to the eye to follow the current flow direction.

our calculation showed that nanotori formed from the metal-1 zigzag nanotube $(9,0)$ consisting of 1332 and 1296 atoms have large magnetic moments of $75.6 \mu_{B}$ and $73.6 \mu_{B}$, respectively. These magnetic moments correspond to a value of $\sim 26.4 \mathrm{emu} / \mathrm{g}$, compared to a value of $-30 \times 10^{-3} \mathrm{emu} / \mathrm{g}$ for the graphite under an applied field of $0.1 \mathrm{~T}$ along the $c$ axis. Thus, the nanotori $(9,0) / 1332$ and $(9,0) / 1296$ possess giant paramagnetic moments as their strength is 3 orders of magnitude stronger than that of the diamagnetic moment of graphite at $0.1 \mathrm{~T}$. (ii) Nanotori formed from metal-2 nanotubes (i.e., those with the band degeneracy at $k= \pm 2 \pi / 3 T$ [11]) exhibit a giant paramagnetic moment at $0 \mathrm{~K}$ for selected circumferences or magic radii. For example, the $(5,5) / 1500$ nanotorus possesses a large magnetic moment of $88.4 \mu_{B}$ at $0 \mathrm{~K}$. Similarly, the nanotorus $(7,4) / 1860$ has a giant magnetic moment of $98.5 \mu_{B}$ at $0 \mathrm{~K}$. (iii) The paramagnetic moments of the above-mentioned nanotori show a very sensitive dependence on the temperature as their strength decreases by approximately 3 orders of magnitude [from $88.4 \mu_{B}$ to $0.11 \mu_{B}$ for the $(5,5) / 1500$ nanotorus and from $98.5 \mu_{B}$ to $0.12 \mu_{B}$ for the $(7,4) / 1860$ nanotorus] when the temperature increases from 0 to 300 K. (iv) Nanotori formed from metal-2 nanotubes but with radii other than the magic radii exhibit a diamagnetic response with their magnetic moments much weaker than the strength of the paramagnetic moment exhibited by nanotori corresponding to the same metallic nanotube but with magic radii. For example, the $(5,5) / 1480$ nanotorus has a diamagnetic moment of $-0.11 \mu_{B}$ and the $(7,4) / 1364$ nanotorus a magnetic moment of $-0.064 \mu_{B}$. Their respective magnetic moment values also show only a relatively weak dependence on the temperature. (v) Nanotori formed from semiconducting nanotubes exhibit diamagnetic moments that are weaker than those of nanotori formed from metallic nanotubes but exhibiting a diamagnetic moment. The strength of the magnetic moment in these cases is independent of the temperature in the range from 0 to $500 \mathrm{~K}$.

The pattern of behavior of the magnetic responses of nanotori to an applied magnetic field perpendicular to its plane is diversified and full of surprises. Key questions underpinning the physics of this behavior pattern include: (1) Why do nanotori formed from a certain metallic nanotube respond very differently to an applied magnetic field depending on whether they are formed from a metal-1 or a metal-2 nanotube, and why do some of them possess giant magnetic moments? (2) What is the physics responsible for the existence of the magic radii for nanotori corresponding to metal-2 nanotubes, and why do these nanotori possess giant paramagnetic moments? (3) Why do nanotori formed from semiconducting nanotubes always exhibit a diamagnetic response regardless of their radii? (4) Why do the magnetic moments show different temperature behaviors for nanotori of different types?

The answers to these questions can be traced to the interplay between the electronic structure of the nanotube and the geometrical structure of the nanotorus. For a metallic nanotube, its Fermi wavelength, $\lambda_{F}$, is related to its translation vector $T$ by $\lambda_{F}=r T$, where $r=1$ for metal-1 and $r=3$ for metal-2 nanotubes, respectively [11]. If the circumference of a nanotorus, $L=p T$, corresponding to a given metallic nanotube satisfies $L=q \lambda_{F}$ or $p=r q$ where $q$ is an integer, then that nanotorus is metallic. Otherwise, it is a small-gap semiconductor. For nanotori formed from metal-1 nanotubes, the condition $p=q$ is 
satisfied by any integer value of $p$ so that nanotori formed from metal-1 nanotubes are always metallic regardless of their circumference. The two nanotori $(9,0) / 1332$ and $(9,0) / 1296$ shown in Fig. 1 are metallic nanotori because the $(9,0)$ tube is a metal-1 nanotube. On the other hand, for the two nanotori $(5,5) / 1500$ and $(5,5) / 1480$ formed from the metal-2 $(5,5)$ nanotube, $p=75$ and $p=74$, respectively. Clearly, the condition $p=3 q$ is met for the $(5,5) / 1500$ torus but not for the $(5,5) / 1480$ torus (as $p$ is not divisible by 3 ). Therefore, the $(5,5) / 1500$ nanotorus is a metal, while the $(5,5) / 1480$ nanotorus is a small-gap semiconductor. Similarly, the $(7,4) / 1860$ nanotorus is a metal while the $(7,4) / 1364$ nanotorus is a small-gap semiconductor. Thus, our calculations show that a metallic nanotorus will give rise to a giant paramagnetic moment while a semiconducting nanotorus will yield a diamagnetic moment. Our calculations suggest the existence of the magic radii (as dictated by the relation $p=3 q$ ) for nanotori formed from metal-2 nanotubes, while no such selection rule applies to nanotori formed from metal-1 nanotubes.

A simple scenario can be put forth to explain the unusual paramagnetic response of metallic nanotori. In a metallic nanotorus, electrons move freely along the circumference of the torus in two opposite directions because the corresponding nanotube is a ballistic conductor. The associated magnetic dipole moments of the electrons moving along the circumference exactly cancel out when there is no applied magnetic field. With the application of a magnetic field, however small, perpendicular to its plane, the dipole moments will be lined up along the direction of the applied field at low temperatures, yielding a large paramagnetic moment. This scenario is well described by the detailed structure of the ring current in the metallic $(5,5) / 1200$ nanotorus shown in Fig. 2(a). From this figure, it can be seen that the ring current flows along the chiral pattern of the $(5,5)$ nanotube, with its direction consistent with the applied field that is perpendicular to the plane of the torus and points outward. It is this ring current, together with the area enclosed by the torus, that leads to the large paramagnetic moment. Also shown in Fig. 2(b) is the structure and the ring current of the small-gap semiconducting $(7,4) / 1364$ nanotorus. The net current in this case flows in the direction opposite to the direction dictated by the applied field, leading to a diamagnetic response.

To shed light on the mechanisms of the magnetic responses of nanotori, we calculated the free energy $F=$ $-k T \Sigma_{n} \ln \left\{1+\exp \left[-\left(E_{n}-\mu\right) / k T\right]\right\}+N \mu$ of nanotori corresponding to metal-1 and metal-2 nanotubes (with magic as well as nonmagic radii), as well as nanotori corresponding to semiconducting nanotubes as a function of the temperature at various applied fields. Here $E_{n}$ denotes the eigenenergy of the nanotorus in an applied field $B$ and $\mu$ the chemical potential. The magnetic moment of a nanotorus was determined by

$$
M=-\left(\frac{\partial F(B, T)}{\partial B}\right)_{B=0}-B\left(\frac{\partial^{2} F(B, T)}{\partial B^{2}}\right)_{B=0} .
$$

In this expression, the first term takes into account the magnetic moments associated with the ballistic motion of the electrons in the nanotorus while the diamagnetism associated with the tendency of electrons to at least partially shield the interior of the torus from the influence of the applied field is included in the second term. Using Eq. (1), the magnetic moments for the entire set of nanotori considered previously are calculated and the results corresponding to $B=0.1$ and $B=0.2 \mathrm{~T}$ are shown in Fig. 1 as open circles and diamonds, respectively. It can be seen that the agreement between the results of these two independent calculations is excellent as the results based on Eq. (1) fall right on the respective curves obtained from the ring current calculation. It should also be noted that the calculations based on Eq. (1) yielded very small but non-negligible diamagnetic moments for nanotori corresponding to semiconducting nanotubes (see Fig. 1). The comparison between the two independent calculations clearly indicates that the magnetic response of a nanotorus is determined by the combined effect of the magnetic moments associated with the ballistic motion of the electrons in the torus and the diamagnetic response of electrons.

For metallic nanotori at $T=0 \mathrm{~K}$, Eq. (1) yields $M \approx M_{m}+\chi B$, where $M_{m}=4 m=-4\left(\partial E_{m} / \partial B\right)_{B=0}$ with $E_{m}$ being the energy of the highest occupied states and $\chi=-\sum_{n}^{\text {occ }}\left(\partial^{2} E_{n} / \partial B^{2}\right)_{B=0}$. It should be noted that $m$ may be construed as the dipole moment associated with the ballistic motion of the electron along the nanotorus. The factor 4 is due to the fact that there are four electrons occupying the highest occupied states. For $k T \gg m B$, Eq. (1) leads to $M \approx$ $\left[\chi+\left(M_{m}^{2} / 8 k T\right)\right] B$. For semiconducting nanotori, Eq. (1) gives rise to $M=\chi B$ at $T=0 \mathrm{~K}$, while it yields $M \approx\left\{\chi+\left[e^{-(\Delta / k T)} /\left(1+e^{-(\Delta / k T)}\right)^{2}\right]\left(M_{m}^{2} / 2 k T\right)\right\} B$ in the vicinity of the room temperature with $\Delta \approx E_{G} / 2$ and $E_{G}$ being the energy gap. In the case of $(5,5) /$ $1500, M_{m}=5.162 \times 10^{-3} \mathrm{eV} / \mathrm{T}=89.15 \mu_{B}$ and $\chi=$ $-6.96 \times 10^{-5} \mathrm{eV} / \mathrm{T}^{2}$ (compared to a value of $-5.606 \times$ $10^{-5} \mathrm{eV} / \mathrm{T}^{2}$ for a 1500 -atom graphite). For an applied field of $0.1 \mathrm{~T}, M_{m}$ is 3 orders of magnitude greater than the diamagnetic moment of $-6.96 \times 10^{-6} \mathrm{eV} / \mathrm{T}$ or $-0.12 \mu_{B}$, yielding a large paramagnetic moment of $89.03 \mu_{B}$ at $0 \mathrm{~K}$. At $300 \mathrm{~K}$, these values of $M_{m}$ and $\chi$ lead to a magnetic moment of $6.15 \times 10^{-6} \mathrm{eV} / \mathrm{T}$ or $0.106 \mu_{B}$, a decrease of 3 orders of magnitude from its value at $0 \mathrm{~K}$. For the small-gap semiconducting $(5,5) / 1480$ nanotorus, $M_{m}=5.14 \times 10^{-3} \mathrm{eV} / \mathrm{T}, \quad \chi=-6.67 \times 10^{-5} \mathrm{eV} / \mathrm{T}^{2}$, and $E_{G}=0.13 \mathrm{eV}$. At $0 \mathrm{~K}$, it exhibits a diamagnetic moment $M=\chi B=-0.115 \mu_{B}$. But because its $\Delta$ value is of the same order of magnitude as $k T$ at $300 \mathrm{~K}$, the strength of its magnetic moment is reduced only by about a factor of 2 . For the semiconducting $(10,0) / 1600$ 
nanotorus, $\quad M_{m}<10^{-9} \mathrm{eV} / \mathrm{T}, \quad \chi=-6.96 \times$ $10^{-5} \mathrm{eV} / \mathrm{T}^{2}$, and $E_{G}=0.95 \mathrm{eV}$. Therefore, it exhibits a very small diamagnetic moment. In addition, this diamagnetic moment is insensitive to a temperature up to $500 \mathrm{~K}$ as $\Delta \gg k T$ in this case. Thus, our analysis based on the consideration of the free energy [Eq. (1)] not only confirmed the results of the ring current calculation but also elaborated the underlying physics for the magnetic response of a nanotorus to an applied field perpendicular to its plane. Specifically, the magnetic response of a nanotorus is determined by the combination of the paramagnetic moment of the ballistic motion of the electrons in the torus and the diamagnetic response.

Since $M=I \sigma$, the paramagnetic moment of a metallic nanotorus is expected to vary linearly with the radius. This relationship is confirmed by our numerical calculation of paramagnetic moments of metallic nanotori. For example, we find that $M=296.8 \mu_{B}$ for $(5,5) / 5100$ and $M=88.4 \mu_{B}$ for $(5,5) / 1500$. Similarly, we find that $M=$ $304.3 \mu_{B}$ for $(7,4) / 5580$ and $M=98.5 \mu_{B}$ for $(7,4) / 1860$. Since the radii of the experimentally observed nanotori, (ranging from 150 to $270 \mathrm{~nm}$ ), are about 2 orders of magnitude larger [7-10] than metallic nanotori considered in the present work, it is not too difficult to contemplate metallic nanotori with a colossal paramagnetic moment of about $10^{4} \mu_{B}$ at low temperatures as soon as a magnetic field is switched on. The realization of nanoscale structures possessing such unusual magnetic properties will undoubtedly bring about unexpected as well as expected applications in the molecular scale device arena.

Questions can be raised about the possible effect of the curvature of the nanotube on the magnetic response of metallic nanotori predicted by the $\pi$-orbital tightbinding theory. For armchair nanotubes, neither the curvature nor the Peierls instability affect the metallic condition (zero gap) [11,15-17]. Hence, our prediction about the magnetic response of armchair nanotubes should remain valid. For nanotubes $\left(n_{1}, n_{2}\right)$, with $n_{1}-n_{2}=3 n$ and $n \neq 0$, both theoretical calculations $[16,18,19]$ and experimental measurements $[17,20]$ have shown that they are quasimetallic because the effect of the curvature leads to a pseudoenergy gap of $\sim 10 \mathrm{meV}$. With an energy gap, the magnetic response is dictated by the delicate balance between $m B$ and $\Delta=E_{G} / 2$. If $m B>\Delta$ and for $m B-\Delta \gg k T, M \approx 4 m+\chi B$ according to Eq. (1). The magnetic moment $m$ is about $1.07 \times 10^{-3} \mathrm{eV} / \mathrm{T}$ for the quasimetallic nanotorus $(7,4) / 1364$ and about $1.10 \times 10^{-3} \mathrm{eV} / \mathrm{T}$ for the $(9,0) / 1332$ nanotorus. With radii of experimentally observed nanotori being about 2 orders of magnitude greater than those of nanotori with 1500 atoms, $m$ of quasimetallic nanotori of these sizes is expected to be $\sim 10^{-1} \mathrm{eV} / \mathrm{T}$. The condition $m B>\Delta$ can then be easily satisfied by a magnetic field of $0.1 \mathrm{~T}$, leading to a paramagnetic moment at least 3 orders of magnitude greater than the diamagnetic moment of graphite. Hence, large paramagnetic response can be realized for quasimetallic nanotori with very small pseudoenergy gaps due to the same interplay between the geometric structure of nanotori and the ballistic motion of the electron.

We have also considered the effects due to mechanical deformation and chemical defect, and the preliminary results indicate that these effects are small. Therefore, the unusual magnetic response of nanotori reported here is very robust. Although we have not studied polygon-shaped toroidal structures dictated by 5-7 defect pairs, we believe that, as long as there are only a sufficiently small number of such defect pairs so that the ballistic nature of the electrons are not affected, the polygon-shaped nanotori may also exhibit large paramagnetic response.

Finally, we remark that, while the two-dimensionally polymerized rhombohedral $\mathrm{C}_{60}$ was most recently observed to exhibit a paramagnetic response [21], our theoretical prediction of the colossal paramagnetic response of metallic carbon nanotori provides a unique example of the unusual magnetic behavior of carbon based nanostructures.

This work was supported by the NSF (Grants No. DMR-011284 and No. DMR-9802274), the U.S. DOE (DE-FG02-00ER45832), and the National Science Council of Taiwan.

[1] H. W. Kroto et al., Nature (London) 318, 162 (1985).

[2] V. Elser and R.C. Haddon, Nature (London) 325, 792 (1987).

[3] R. C. Haddon et al., Nature (London) 350, 46 (1991).

[4] R. S. Ruoff et al., J. Phys. Chem. 95, 3457 (1991).

[5] A. P. Ramirez et al., Science 265, 84 (1994).

[6] J. Heremans, C. H. Olk, and D. T. Morelli, Phys. Rev. B 49, 15122 (1994).

[7] J. Liu et al., Nature (London) 385, 780 (1997).

[8] M. Ahlskog et al., Chem. Phys. Lett. 300, 202 (1999).

[9] M. Sano et al., Science 293, 1299 (2001).

[10] R. Martel, H. R. Shea, and P. Avouris, J. Phys. Chem. 103, 7551 (1999).

[11] R. Saito, G. Dresselhaus, and M. S. Dresselhaus, Physical Properties of Carbon Nanotubes (Imperial College, London, 1998).

[12] J. P. Lu, Phys. Rev. Lett. 74, 1123 (1995).

[13] M. Graf and P. Vogl, Phys. Rev. B 51, 4940 (1995).

[14] T. B. Boykin, R. C. Bowen, and G. Klimeck, Phys. Rev. B 63, 245314 (2001).

[15] J. W. Mintmire, B. I. Dunlap, and C. T. White, Phys. Rev. Lett. 68, 631 (1992).

[16] C. Kane and E. Mele, Phys. Rev. Lett. 78, 1932 (1997).

[17] M. Ouyang et al., Science 292, 702 (2001).

[18] X. Blase et al., Phys. Rev. Lett. 72, 1878 (1994).

[19] C. White et al., in Clusters and Nanostructured Materials, edited by P. Jena and S. N. Behera (Nova, New York, 1996), p. 231.

[20] C. Zhou, J. Kong, and H. Dai, Phys. Rev. Lett. 84, 5604 (2000).

[21] T. L. Makarova et al., Nature (London) 413, 716 (2001). 